\title{
Shunting versus Inactivation: Analysis of Presynaptic Inhibitory Mechanisms in Primary Afferents of the Crayfish
}

\author{
Daniel Cattaert ${ }^{1}$ and Abdeljabbar El Manira ${ }^{2}$ \\ 1 Laboratoire Neurobiologie et Mouvements, Centre National de la Recherche Scientifique, 13402 Marseille Cedex 20, \\ France, and 2 The Nobel Institute for Neurophysiology, Department of Neuroscience, Karolinska Institutet, S-171 77 \\ Stockholm, Sweden
}

Primary afferent depolarizations (PADs) are associated with presynaptic inhibition in both vertebrates and invertebrates. In the present study, we have used both anatomical and electrophysiological techniques to analyze the relative importance of shunting mechanisms versus sodium channel inactivation in mediating the decrease of action potential amplitude, and thereby presynaptic inhibition. Experiments were performed in sensory afferents of a stretch receptor in an in vitro preparation of the crayfish. Lucifer yellow intracellular labeling of sensory axons combined with GABA immunohistochemistry revealed close appositions between GABA-immunoreactive (ir) fibers and sensory axons. Most contacts were located on the main axon at the entry zone of the ganglion, close to the first branching point within the ganglion. By comparison, the output synapses of sensory afferents to target neurons were located on distal branches. The location of synaptic inputs mediating spontaneous PADs was also determined electrophysiologically by making dual intracellular recordings from single sensory axons. Inputs generating PADs appear to occur around the first axonal branching point, in agreement with the anatomical data. In this region, small PADs (3-15 $\mathrm{mV}$ ) produced a marked reduction of action potential amplitude, whereas depolarization of the membrane potential by current injection up to $15 \mathrm{mV}$ had no effect. These results suggest that the decrease of the amplitude of action potentials by single PADs results from a shunting mechanism but does not seem to involve inactivation of sodium channels. Our results also suggest that GABAergic presynaptic inhibition may act as a global control mechanism to block transmission through certain reflex pathways.

Key words: presynaptic inhibition; primary afferent depolarization; crayfish; synaptic transmission; chloride conductance; sodium channels inactivation
Presynaptic inhibition of sensory transmission is associated with primary afferent depolarizations (PADs) (Clarac et al., 1992; Nusbaum et al., 1997; Rudomin et al., 1998). During locomotion in vertebrates, sensory afferents terminals receive phasic PADs that can trigger antidromic action potentials (Gossard et al., 1989, 1990). These PADs are likely to involve the activation of $\mathrm{GABA}_{\mathrm{A}}$ receptors that increase the conductance to chloride, which has an equilibrium potential around $-30 \mathrm{mV}$ (Allen and Burnstock, 1990). In the crayfish locomotor system, PADs decrease the amplitude of the afferent action potentials, thereby depressing monosynaptic EPSPs in target neurons (Cattaert et al., 1992). A similar mechanism occurs in sensory afferents in the locust (Burrows and Matheson, 1994). In crayfish, PADs elicit antidromic action potentials that do not elicit any postsynaptic effects (El Manira et al., 1991; Cattaert et al., 1992).

The study of presynaptic inhibitory mechanisms in the primary afferents of mammals is difficult to achieve directly using intracellular recordings because of the very small diameter of the afferent axons $(0.5-1.0 \mu \mathrm{m})$ and boutons $(3-6 \mu \mathrm{m})$ where PADs are likely to be produced. Most of the data are derived from anatomical observations (Fyffe and Light, 1984; Maxwell et al.,

\footnotetext{
Received Nov. 30, 1998; revised April 19, 1999; accepted April 27, 1999.

This work was supported by Centre National de la Recherche Scientifique (D.C.), by the Swedish Medical Research Council project 11562 (A.E.), and by the Sweden/ France exchange program. We thank Drs. F. Clarac, D. Parker, P. Wallén, and L. Vinay for their comments on this manuscript.

Correspondence should be addressed to D. Cattaert, Laboratoire Neurobiologie des Réseaux, Centre National de la Recherche Scientifique, UMR 5816, Université de Bordeaux 1, Bat B2, Avenue des Facultés, 33401 Talence Cedex, France. Copyright (C) 1999 Society for Neuroscience $0270-6474 / 99 / 196079-11 \$ 05.00 / 0$
}

1990), measurements of excitability changes by using extracellular electrodes (Rudomin et al., 1998; Lomeli et al., 1998), and intraaxonal recordings of PADs at a long distance from the axoaxonic synapse (Jimenez et al., 1988). Two mechanisms, based on simulation studies, have been proposed to account for presynaptic inhibition associated with PADs: a shunting mechanism (Segev, 1990) and inactivation of sodium channels (Graham and Redman, 1994). In mammals, however, experimental evidence for either of these mechanisms is still lacking.

The crayfish locomotor system provides a convenient experimental model to analyze the relative importance of these two mechanisms in presynaptic inhibition as a result of PADs. The three phenomena associated with PADs and with presynaptic inhibition in vertebrate (depolarization, increase of chloride conductance, and antidromic spikes) are also present in the primary afferents of a chordotonal organ in the crayfish (Cattaert et al., 1992). In these afferents, spontaneous PADs occur either randomly in quiescent preparations or at a fixed phase of the locomotor cycle in preparations displaying locomotor activity (El Manira et al., 1991; Cattaert et al., 1992). The possibility of recording intracellularly from the regions where the GABA synapses that produce PADs are located offers a significant advantage in the analysis of the mechanisms of presynaptic inhibition in this invertebrate system. In this paper, using electrophysiological and immunohistochemical techniques, we have localized the region of the sensory axon where these spontaneous PADs are produced and have determined the effect of PADs on spike conduction. Our findings suggest that PADs mediate their inhibitory effects mainly through shunting mechanisms, likely occur- 
Figure 1. Experimental setup. A, The in vitro preparation of the crayfish thoracic locomotor system consisting of ganglia 3-5 (G3, G4, G5) dissected together with motor nerves of the proximal muscles and the coxo-basal chordotonal organ $(C B C O)$. B, Enlargement of the region indicated by the dashed-line box showing a sensory axon terminal and postsynaptic motoneurons in the ganglion. $M E 1$, Proximal electrode; $M E 2$, distal electrode. The terms proximal and distal will always refer to this relative disposition. Distal processes of a sensory terminal are closer to the motoneurons $(M N)$. $C$, Staining of all CBTs in the left 5th ganglion of crayfish. The localization of the CBTs within the ganglion is represented in C1. Most of the CBTs have the same anatomy, and all of them are more or less superimposed (2). A single CBT on which GABA-ir boutons were found was reconstructed from slices (3). The box represents the zone in which close appositions between sensory axons and GABA-ir fibers were found (Fig. 2A).

ring on the main axon of the sensory neuron arborization, at some distance from output synapses. In such an organization, GABAergic PADs would therefore inhibit synaptic transmission in all output synapses and do not seem to mediate a selective control at different branches of single sensory axons.

\section{MATERIALS AND METHODS}

Experimental animals. Experiments were performed on male and female crayfish (Procambarus clarkii; $n=14$ ) weighing 25-30 gm. The animals were purchased from a commercial supplier (Chateau Garreau, Landes, France) and kept in circulating fresh water at $18-20^{\circ} \mathrm{C}$.

In vitro preparation. An in vitro preparation of the thoracic locomotor nervous system was used as described previously (Sillar and Skorupski, 1986; Chrachri and Clarac, 1989). This preparation consists of the last three thoracic ganglia (3-5) along with the motor nerves from the fifth ganglion to the promotor, remotor, levator, and depressor muscles (Fig. $1 A)$. The coxo-basal chordotonal organ ( $\mathrm{CBCO}$ ) stretch receptor was also dissected and kept intact. The preparation was pinned with the dorsal side up in a silicone elastomer (Sylgard)-lined Petri dish. The fourth and fifth ganglia were desheathed to improve the superfusion of the central neurons and allow intracellular recordings from $\mathrm{CBCO}$ axon terminals (CBTs) (Fig. 1B). The nervous system was continuously superfused with oxygenated control saline containing (in mM): $195 \mathrm{NaCl}, 5.5$ $\mathrm{KCl}, 13.5 \mathrm{CaCl}_{2}, 2.5 \mathrm{MgCl}_{2}$, and 10 Tris, at $\mathrm{pH}$ 7.6.
Electrodes and recordings. Sensory nerve activity was recorded with platinum "en-passant" electrodes connected to homemade amplifiers (gain $\times 10,000-100,000$ ). Intracellular recordings from CBTs within the ganglion (Fig. $1 B$ ) were performed with micropipettes filled with either $\mathrm{K}$-acetate $(2 \mathrm{M}, 30-40 \mathrm{M} \Omega)$ or Lucifer yellow $(5 \%$ in $3 \mathrm{M} \mathrm{LiCl}, 60-100$ $\mathrm{M} \Omega$ ). An Axoclamp 2A amplifier (Axon Instruments, Foster City, CA) was used in current-clamp mode, with K-acetate-filled electrodes, and the level of saline was as low as possible to reduce the micropipette capacitance. CBTs were identified on the basis of two criteria. First, injection of depolarizing current pulses elicited spikes in CBTs that were correlated in a one-to-one manner with extracellular spikes recorded in the $\mathrm{CBCO}$ sensory nerve. Second, orthodromic spikes produced by the $\mathrm{CBCO}$ sensory neurons were correlated with intracellular spikes at fixed time in CBTs. The CBTs analyzed in this study fired action potentials attributable to the existence of spontaneous activity in $\mathrm{CBCO}$ sensory neurons. An eight-channel stimulator (A.M.P.I., Jerusalem, Israel) was used to trigger intracellular pulses in CBTs during the identification procedure. Data were displayed and printed on a four-channel digital oscilloscope (Yokogawa, Tokyo, Japan) and stored on tape (Biological DTR 1800, Claix, France). Results are based on recordings from 16 identified CBTs (11 dual and 5 single intracellular recordings).

Immunohistochemistry. Populations of sensory axons were labeled with Lucifer yellow (Fig. 1C) using the following procedure. The CBCO sensory nerve was cut and placed in a concentrated solution of Lucifer yellow (potassium salt) for $12-18 \mathrm{hr}$ at $5^{\circ} \mathrm{C}$. The preparation was then 

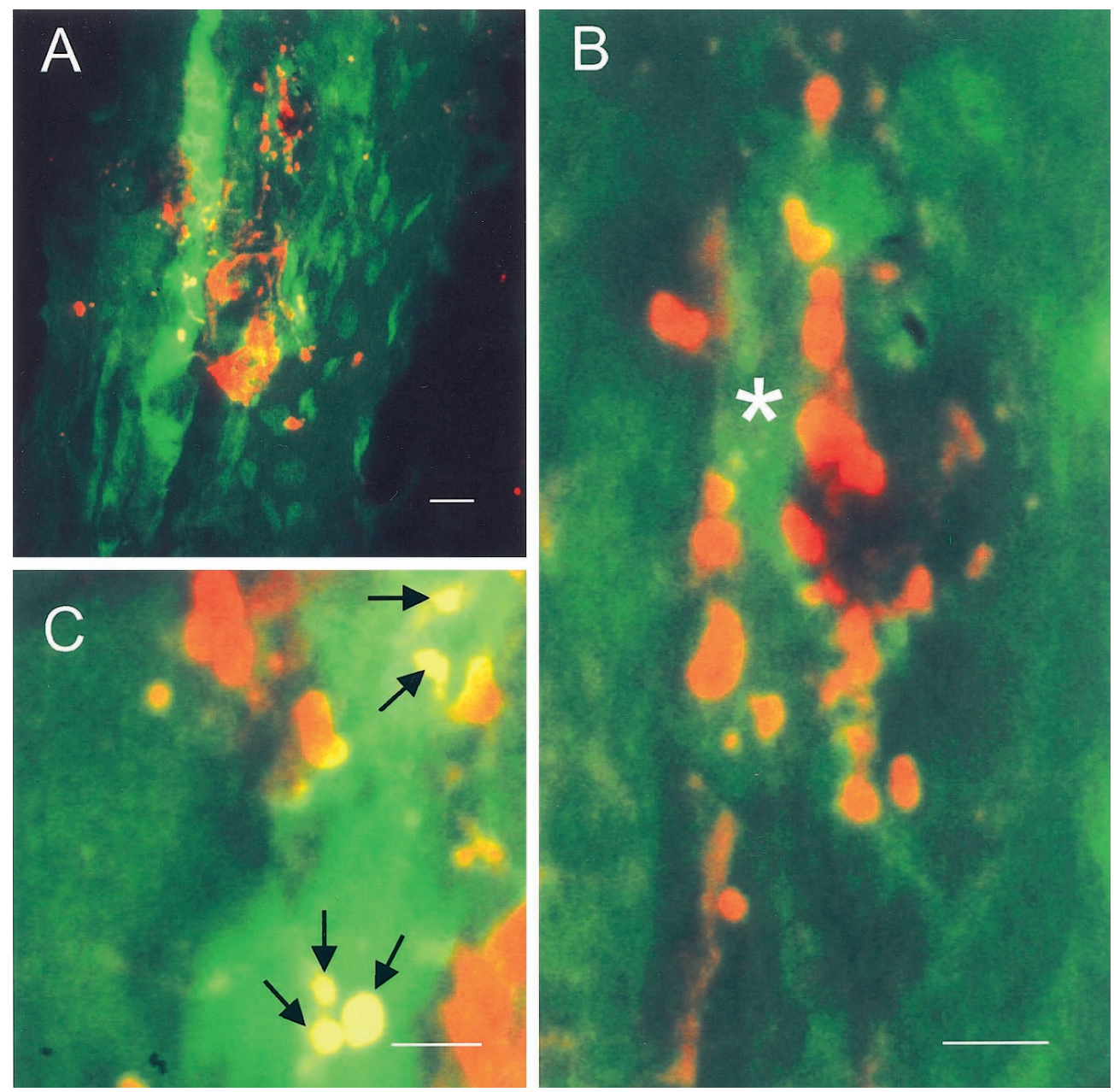

Figure 2. Localization of GABA-immunoreactive boutons on Lucifer yellowfilled CBCO sensory axons, using confocal microscope analysis of a $30-\mu \mathrm{m}$-thick slice. Only projection views are given. $A$, Global view of the slice showing $\mathrm{CBCO}$ sensory axons backfilled with Lucifer yellow (green) and GABA-immunoreactive structures (red). Scale bar, $5 \mu \mathrm{m}$. B, Details of GABA-immunoreactive boutons particularly abundant in the vicinity of a $\mathrm{CBCO}$ axon (asterisk) stained by Lucifer yellow. Note, however, that GABAimmunoreactive boutons are aligned and follow the CBCO axon. Scale bar, $2 \mu \mathrm{m}$. $C$, Detailed view of five GABA-immunoreactive boutons (arrows) that are in close apposition with the best stained $\mathrm{CBCO}$ axon. Scale bar, $2 \mu \mathrm{m}$. fixed for $3 \mathrm{hr}$ in a solution containing $2.5 \%$ glutaraldehyde, $1 \%$ paraformaldehyde, and $0.2 \%$ picric acid in a $0.1 \mathrm{M}$ phosphate buffer at $\mathrm{pH} 7.4$, and for $3 \mathrm{hr}$ in an acetic "Bouin" solution containing $250 \mathrm{ml}$ saturated picric acid, $750 \mathrm{ml} \mathrm{37 \%}$ paraformaldehyde, and $50 \mathrm{ml}$ glacial acetic acid. The preparation was then rinsed for $15 \mathrm{~min}$ in a $0.9 \% \mathrm{NaCl}$ solution containing $0.05 \mathrm{~m}$ Tris at $\mathrm{pH}$ 7.6. Slices (30 $\mu \mathrm{m}$ thick) were made, dehydrated in a series of alcohols, and stored in $95 \%$ alcohol.

GABA immunostaining was performed after rehydration of the slices using rabbit monoclonal GABA antibody (Sigma, St. Louis, MO) revealed with a rhodamine tetramethyl-rhodamine-isothiocyanate fluorochrome anti-rabbit antibody conjugate (Sigma). Sections were examined using a confocal microscope.

\section{RESULTS}

\section{Immunohistochemical localization of GABAergic inputs to sensory axons}

We have shown previously that spontaneous PADs occurring in CBTs are blocked by the GABA receptor antagonist picrotoxin and that local application of GABA on the CBTs induces a depolarization of membrane potential associated with a decrease in input resistance that is also blocked by picrotoxin (El Manira and Clarac, 1991; Cattaert et al., 1992). PADs and GABAmediated depolarizations have a similar reversal potential (approximately $-35 \mathrm{mV}$ ) (Cattaert et al., 1992), and their amplitude increases when the chloride reversal potential is shifted toward more depolarized values (El Manira and Clarac, 1991; Cattaert et al., 1994). To determine the location of GABAergic contacts, labeling of CBTs with Lucifer yellow (Fig. 2, green) combined with GABA-immunohistochemistry (Fig. 2, red) was performed.
The preparations $(n=3)$ were scanned using a confocal microscope, and the presence of close appositions between GABA-ir and Lucifer yellow-filled axons was analyzed. GABA-ir processes appeared to surround each CBT (Fig. $2 A$; asterisk in $B$ ), close to the first branching point of the sensory axon in the ganglion (Fig. $1 C$, box). Sites of close apposition appear as yellow spots in the confocal microscopy image. These spots result from a merge of the green and red color within a very small volume in the same focal plane. This indicates that these close appositions between the sensory terminal (in green) and the GABA-ir fibers (in red) presumably correspond to synaptic contacts, although an ultrastructural analysis is required to confirm the presence of synaptic inputs. These appositions were located primarily on the main axon and consisted of five to seven contact points (Fig. 2C, yellow spots, arrows), which are likely to represent the superposition of CBTs and presynaptic terminals of GABAergic fibers. However, some CBTs $(20 \%)$ did not have any close appositions with GABA-ir fibers, suggesting that not all CBT sensory axons receive GABAergic inputs. No close appositions were found on the distal thin processes of CBTs [i.e., sites closer to the motoneurons (MNs)]. These results thus indicate that GABAergic inputs occur close to the first branching point of CBTs in the ganglion.

Zones of possible synaptic contacts between CBCO sensory terminals and target motoneurons were analyzed by performing three-dimensional (3D) reconstruction of pairs of sensory afferents and monosynaptically connected MNs. After physiological 

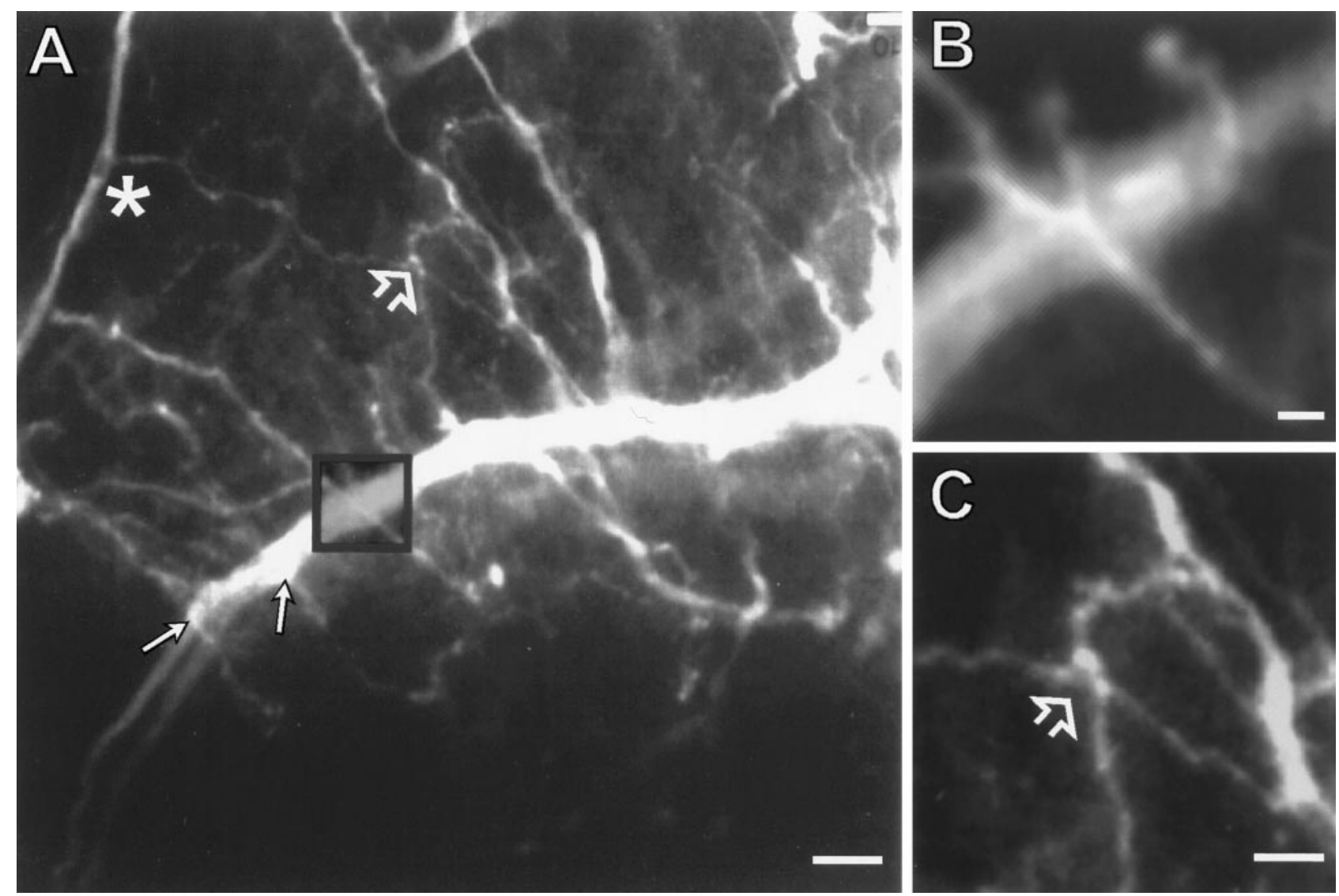

Figure 3. Localization of zones of synaptic contacts between sensory axons and target motoneurons. $A$, A monosynaptically connected CBT and an MN were recorded intracellularly and injected with Lucifer yellow. The two neurons were reconstructed in 3D using a laser-scanning confocal microscope. Zone of close appositions were found after the branching point, both on the main neurite (small arrows) and on small branches of the MN (open arrow). Scale bar, $20 \mu \mathrm{m}$. B. High magnification of a possible zone of close apposition between a sensory process and the main neurite of the MN. Scale bar, $4 \mu \mathrm{m}$. $C$, High magnification of a zone of close apposition between a branch of the sensory axon and a neurite of the MN. Scale bar, $10 \mu \mathrm{m}$.

characterization of the existence of monosynaptic inputs, the pairs of sensory and motor neurons were intracellularly injected with Lucifer yellow (Fig. 3) $(n=3)$. The preparations were fixed, dehydrated, cleared, and subsequently scanned using a confocal microscope. Figure 3A shows a CBT (asterisk) in the ganglion, after the first branching point (not shown in the photograph), and branches from the neurite (thick processes) of a MN. The CBT makes close contacts (i.e., the two processes are in the same focal plane) on both the main (small arrows and large box) and the small branches (open arrow) of the MN. These contacts may correspond to zones of synaptic interaction (Fig. 3B,C). These results suggest that output synapses are located more distally in relation to GABAergic synapses that mediate PADs.

\section{Localization of the transition between active and passive spike propagation in a CBT}

We have demonstrated previously that action potentials are passively conducted in the distal processes of CBCO sensory axons (Cattaert et al., 1992). To determine the site at which action potentials start to be passively propagated, and whether PADs are produced in an area of active or passive conduction, we performed experiments $(n=13)$ with dual intracellular recordings from single CBTs. The distance between the two intracellular recording electrodes was measured through the microscope during the experiment. The location of the recording site was revealed by short injection of Lucifer yellow into the CBT from the most proximal electrode.
The CBTs have a simple morphology that allows a direct comparison of the data obtained in different experiments. The CBTs enter the ganglion, turn rostrally, and give rise to some small lateral branches (Fig. 4A). Overshooting action potentials were recorded intracellularly at axonal sites up to $100 \mu \mathrm{m}$ before the first branching point (amplitude $=76 \pm 3 \mathrm{mV}$ at a resting potential of $-71 \pm 4 \mathrm{mV} ; n=5$ ). The half-spike width of the action potentials was $1.20 \pm 0.05 \mathrm{msec}$. In this area of the CBT (80-100 $\mu \mathrm{m}$ after the first branching point), the spikes appeared to be actively conveyed because no change in their shape (timeto-peak and rising-phase slope, and repolarization phase time) was observed over this distance (Fig. $4 B$ ). A slight reduction of the spike amplitude $(\sim 3 \mathrm{mV})$ and a small conduction delay (150 $\mu \mathrm{sec})$, however, were generally observed ( $n=3$ of 5 ) (Fig. $4 B$ ).

When action potentials were recorded $150 \mu \mathrm{m}$ more distally than the first branching point, their amplitude was smaller, their rising phase was slower, and their half-spike width was wider (1.43 $\pm 0.05 \mathrm{msec}, n=8)$ than that of the same action potential recorded in the region of the first branching point (compare thick and thin traces in Fig. 5B,C). All of these changes were statistically significant ( $p<0.01 ; t$ test). Note that ME1 location in Figure 5 corresponds roughly to ME2 location in Figure 4. The half-spike widths were similar in both recordings $(1.23 \mathrm{msec}$ in Fig. 4, $M E 2$, and $1.22 \mathrm{msec}$ in Fig. 5, ME1), although their amplitude was slightly different (70 mV in Fig. 4, ME2, and $63 \mathrm{mV}$ in Fig. 5, ME1). 

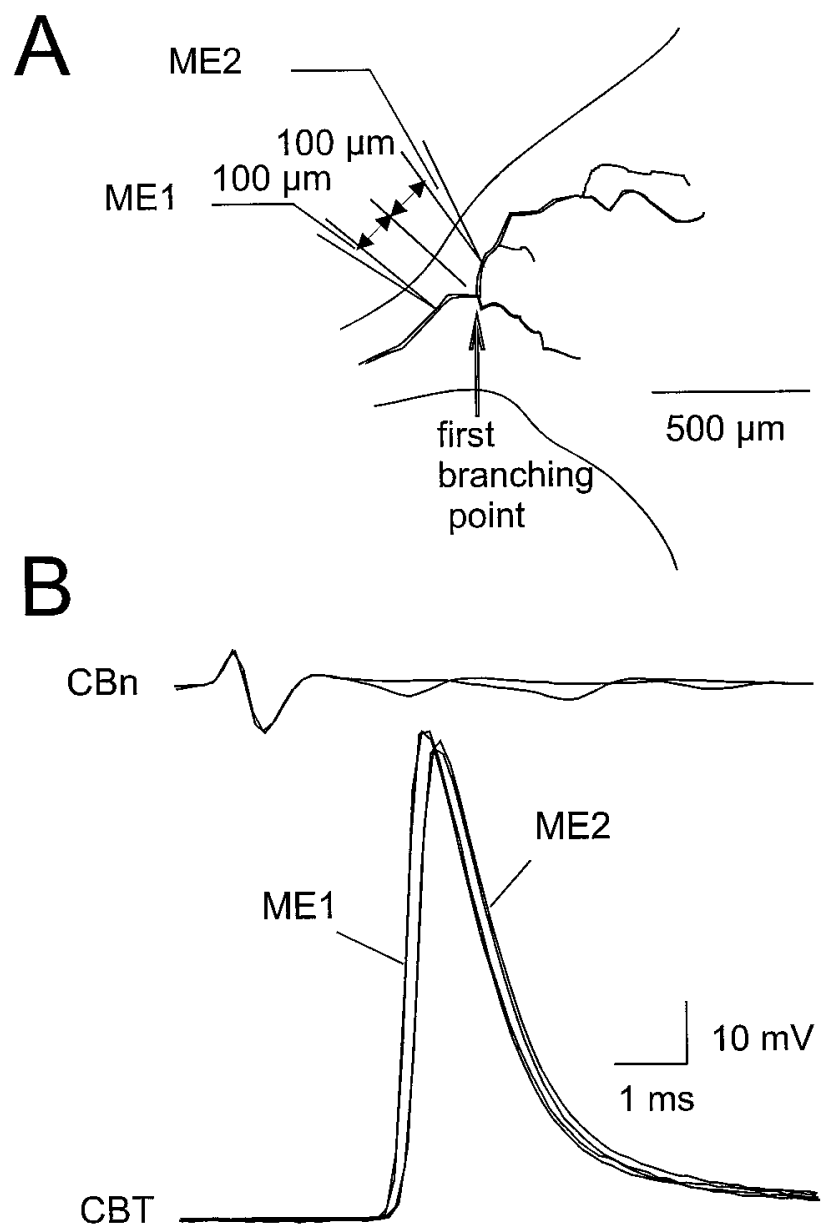

Figure 4. Active propagation of spikes in $\mathrm{CBCO}$ sensory axons in the region of the first branching point. $A$, Schema of the experimental procedure using dual intracellular recordings (ME1, $100 \mu \mathrm{m}$ before the branching point; ME2, $100 \mu \mathrm{m}$ after the branching point) from a single sensory axon in the region of the first branching point (arrow). B, Superimposed intracellular recordings from ME1 and ME2 (CBT, bottom trace) and extracellular recordings from the $\mathrm{CBCO}$ sensory nerve $(\mathrm{CBn}$, top trace).

\section{Electrophysiological localization of GABAergic synaptic input mediating PADs}

GABAergic PADs occur either randomly in quiescent preparations or at a fixed phase of the locomotor cycle in preparations displaying locomotor activity (El Manira et al., 1991; Cattaert et al., 1992). The electrophysiological localization of GABAergic synapses was determined by comparing the amplitudes of spontaneous PADs and their effect on spike amplitude at two intracellular recording sites along the CBT. In the experiment reported in Figure 5, the proximal electrode (ME1) was placed 50 $\mu \mathrm{m}$ after the first branching point (active propagation zone), and the second electrode (ME2) was placed $150 \mu \mathrm{m}$ more distal (passive zone). The amplitude of PADs was slightly larger at ME1 than ME2 (Fig. 5B-D). The reduction of spike amplitude was more pronounced, however, than that of the PADs (Fig. $5 C, D)$. Figure 6 shows the time course of the change of the amplitude of action potentials relative to PADs. The decrease of the amplitude of afferent action potentials displayed a mirror image of PADs, with the maximum reduction occurring at the peak amplitude of PADs (Fig. 6A,B).
The analysis of PAD and spike amplitude is shown in Figure 7. In this experiment, the spike amplitudes ranged from 50 to $63 \mathrm{mV}$ in ME1 and from 40 to $56 \mathrm{mV}$ in ME2. The variation of the spike amplitude was attributable to a continuous discharge of PADs that shunted the spikes (Fig. 5B). The amplitude of the spikes recorded more distally in the branch was linearly related to the amplitude of spikes recorded proximally (Fig. 7A), except for very small spikes (i.e., those that occurred during the largest PADs). The distribution histogram of spike amplitude in both intracellular recording sites had similar shapes, but the distal spike histogram was shifted by $10 \mathrm{mV}$ toward smaller amplitudes (Fig. $7 B)$. The PADs also displayed a large variation in their amplitude ( 0.8 to $4 \mathrm{mV}$ in ME1 and 0.5 to $3.5 \mathrm{mV}$ in ME2). The amplitude of the PADs recorded with ME1 was linearly related to the amplitude of the PADs recorded with ME2 (Fig. 7C), indicating that both electrodes recorded the same events. Most of the points in the diagram are below the $(y=x)$ curve plot (Fig. $7 C)$, showing that the PADs recorded with ME2 had a smaller amplitude than those recorded with ME1 (Fig. 7C,D). To compare the propagation of spikes with that of PADs, we calculated the ratio of the amplitude for PADs and spikes recorded by ME2 with that recorded by ME1. This ratio was constant ( $86 \pm 0.049 \%$ SEM, $n=1000$ ) (Fig. $7 E$ ) for spikes, whereas that of PADs was larger and more variable (92 $\pm 0.84 \%$ SEM, $n=250$ ) (Fig. $7 E$ ), indicating that PADs show less attenuation than spikes (see below).

To determine the location of synaptic inputs mediating PADs in $\mathrm{CB}$ sensory axons, the reduction of the action potential amplitude as they propagated from ME1 to ME2 was compared in the absence and presence of PADs $(n=8)$ (Fig. 8). The two electrodes were positioned after the branching point (same experiments as Fig. 7). In both the absence and presence of PADs, the amplitude of action potentials was reduced to $86 \%$ of the initial value because of the passive propagation between ME1 and ME2 recording sites (Fig. 8A,B). This indicates that no GABAergic synaptic input occurs at regions between ME1 and ME2 (50-100 $\mu \mathrm{m}$ more distal to the branching point), because action potentials were not shunted as they propagated from the ME1 to ME2 recording site (Fig. 8C1,2). Subsequently, the synaptic inputs mediating PADs were likely to occur at regions before the ME1 recording site (Fig. $8 C 1$, around the branching point), and the shunted action potentials would then propagate passively toward the distal processes without being shunted farther. These results, together with those of the GABA immunohistochemistry, show that the synaptic inputs mediating PADs in CBTs are located close to the branching point of the sensory terminals.

\section{Effect of PADs on sodium channel inactivation}

We have shown previously that the GABAergic PADs are caused by an increase in chloride conductance (El Manira and Clarac, 1991; Cattaert et al., 1994), which reduces the peak of action potentials, thereby depressing the amplitude of monosynaptic EPSPs in target neurons (Cattaert et al., 1992). PADs have a reversal potential of between $-35 \mathrm{mV}$ and $-30 \mathrm{mV}$ (Cattaert et al., 1992). During bursts of PADs, it is likely that some $\mathrm{Na}^{+}$ channels could be inactivated, thus reducing the peak of the action potentials. To determine the relative importance of $\mathrm{Na}^{+}$ channels in activation versus shunting mechanisms in causing the decrease of the peak of action potentials during occurrence of PADs, we performed experiments in which intra-axonal recordings were made from sensory afferents either before they reach the ganglion or in a region distal to the first branching point, and 
Figure 5. Comparison of the PAD and spike propagation in passive sites of $\mathrm{CBCO}$ axons. $A$, Diagram of the experimental procedure used. The more proximal microelectrode (ME1) was recorded from a site located just after the first branching point. The more distal microelectrode $(M E 2)$ was recorded $150 \mu \mathrm{m}$ more distally in the main branch. The drawing was reconstructed after Lucifer yellow staining. $B$, Simultaneous intracellular recordings from ME1 and ME2. $C$, Enlarged superimposed ME1 (gray trace) and ME2 (black trace) traces during a spike. The recording of the more distal microelectrode displays a smaller spike with an increased time to peak, therefore indicating that the spike was passively propagated between recordings sites of ME1 and ME2. To make these differences more obvious, the mid-amplitude of both recordings have been aligned (dashed line), and the spike recorded with ME1 has been moved slightly to the right to make both spikes cross the midamplitude line at the same point. $D$, Same arrangement as in $C$ but of a PAD instead of a spike. The amplitude of the distally recorded PAD (ME2, black trace) is slightly smaller than the more proximal recording of the same PAD (ME1, gray trace).

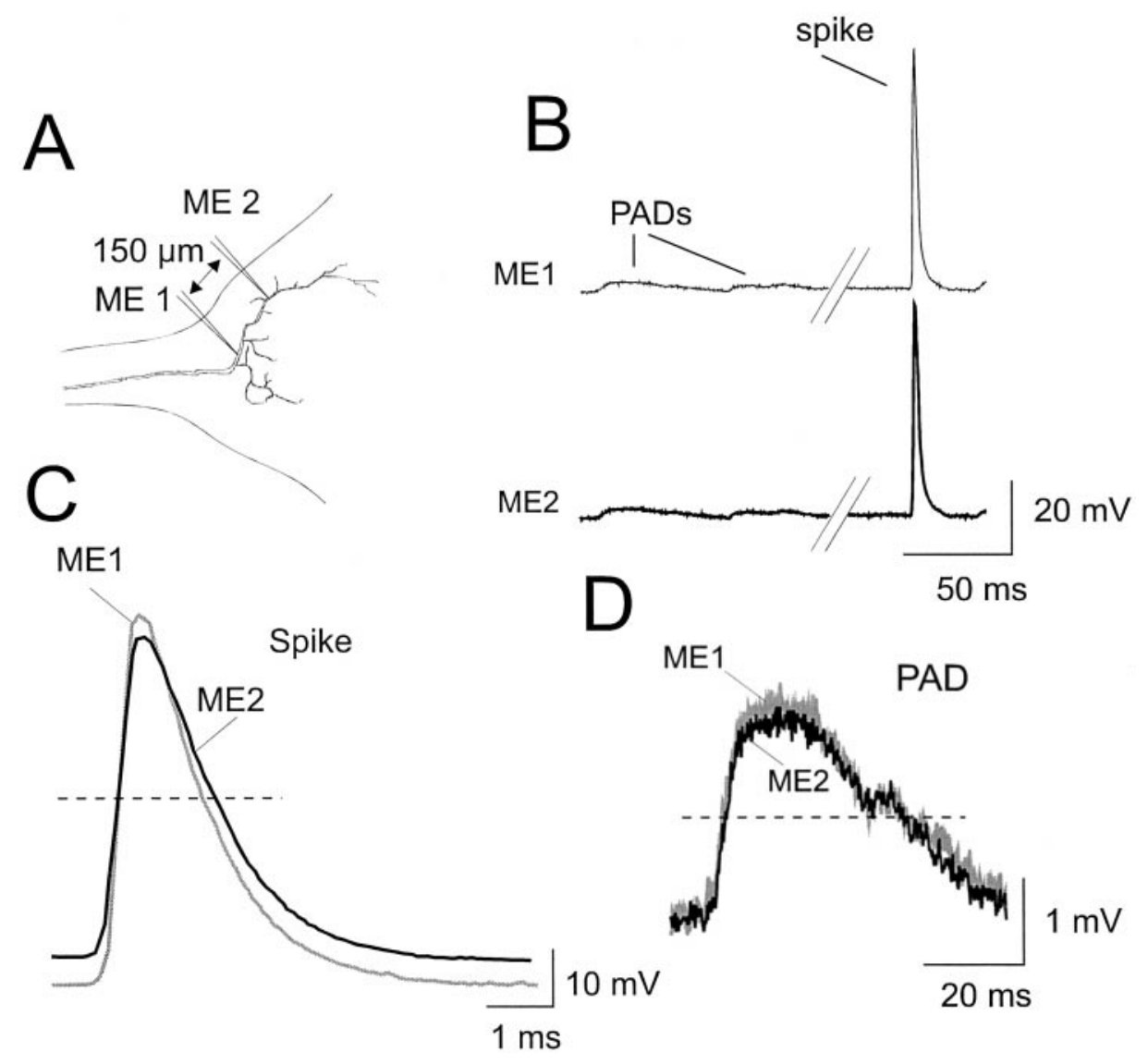

we compared the effects of PADs on the membrane potential and action potential properties (Fig. 9). In experiments $(n=4)$ in which recordings were made from sensory axons before they reach the ganglion, PADs induced depolarization of membrane potential but did not cause any shunting of action potentials. In intracellular experiments in which recordings were made in a region distal to the first branching point (where PADs are produced), PADs produced a depolarization of the membrane potential and shunting of spikes. The results of these experiments are shown in Figure 9. PADs recorded at an axonal site before the sensory nerve reaches the ganglion (Fig. 9A, inset) had no effect on the spike peak (Fig. 9A,B). By contrast, when the recording electrode was situated after $(>100 \mu \mathrm{m})$ the first branching point (Fig. $8 C$, inset), the recorded PADs were able to linearly reduce the spike peak (Fig. 9C,D). The failure of membrane potential depolarization to affect the spike peak suggests that small amplitude $(<10 \mathrm{mV})$ PADs do not cause any inactivation of $\mathrm{Na}^{+}$ channels. However, larger PADs could involve $\mathrm{Na}^{+}$channel inactivation. This question was examined in the following experiments (Fig. 10).

Dual intracellular recordings were performed from single CBTs $(n=5)$ in the region of the first branching point where synaptic inputs mediating PADs are located, and changes in spike peak induced by depolarizing current were compared with those induced by PADs. In the experiment presented in Figure 10, depolarization of the membrane potential of CBTs up to $15 \mathrm{mV}$ above the resting potential did not produce any decrease of the peak of the action potentials. Depolarization of CBTs does not produce any outward rectification. A decrease of the peak of action potentials started to be seen when the CBTs were depo-

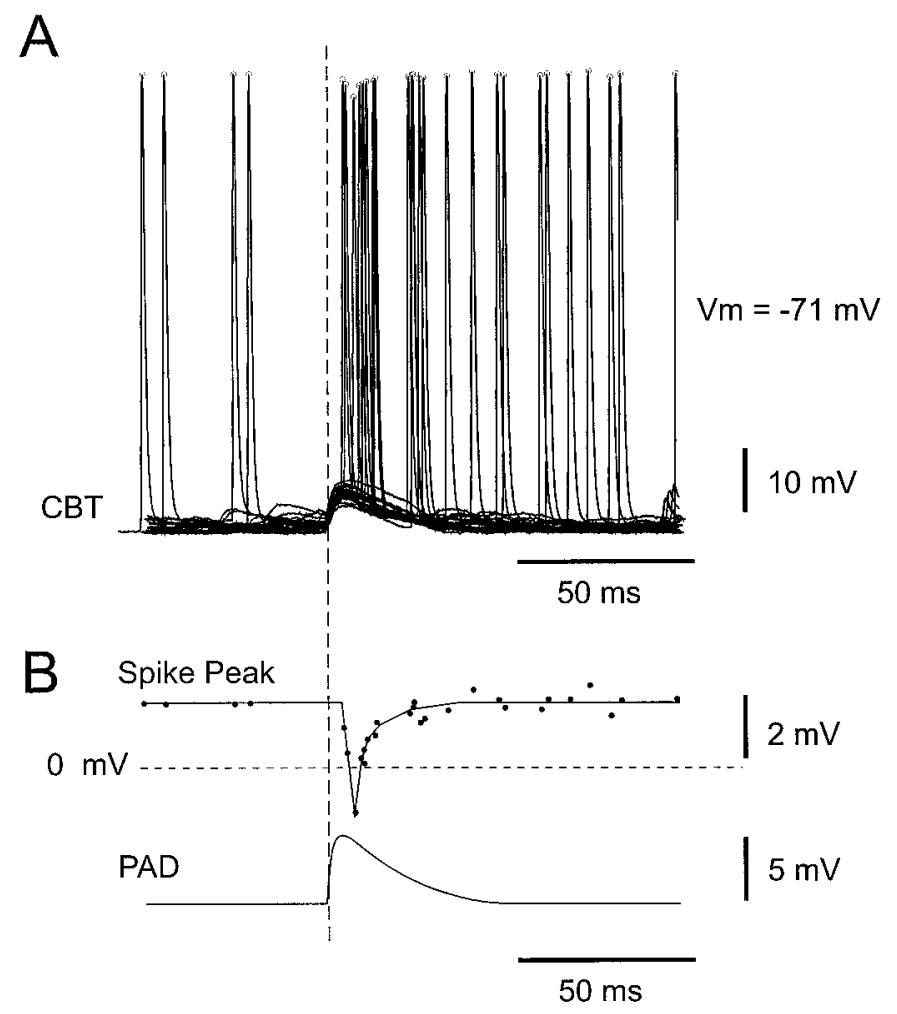

Figure 6. Time course of the decrease of the amplitude of action potential in relation to PADs. $A$, The amplitude of afferent action potentials decreases during the occurrence of PADs. $B$, The maximum decrease in the amplitude of action potentials coincides with the peak of PADs. 


\section{A $\quad B$}
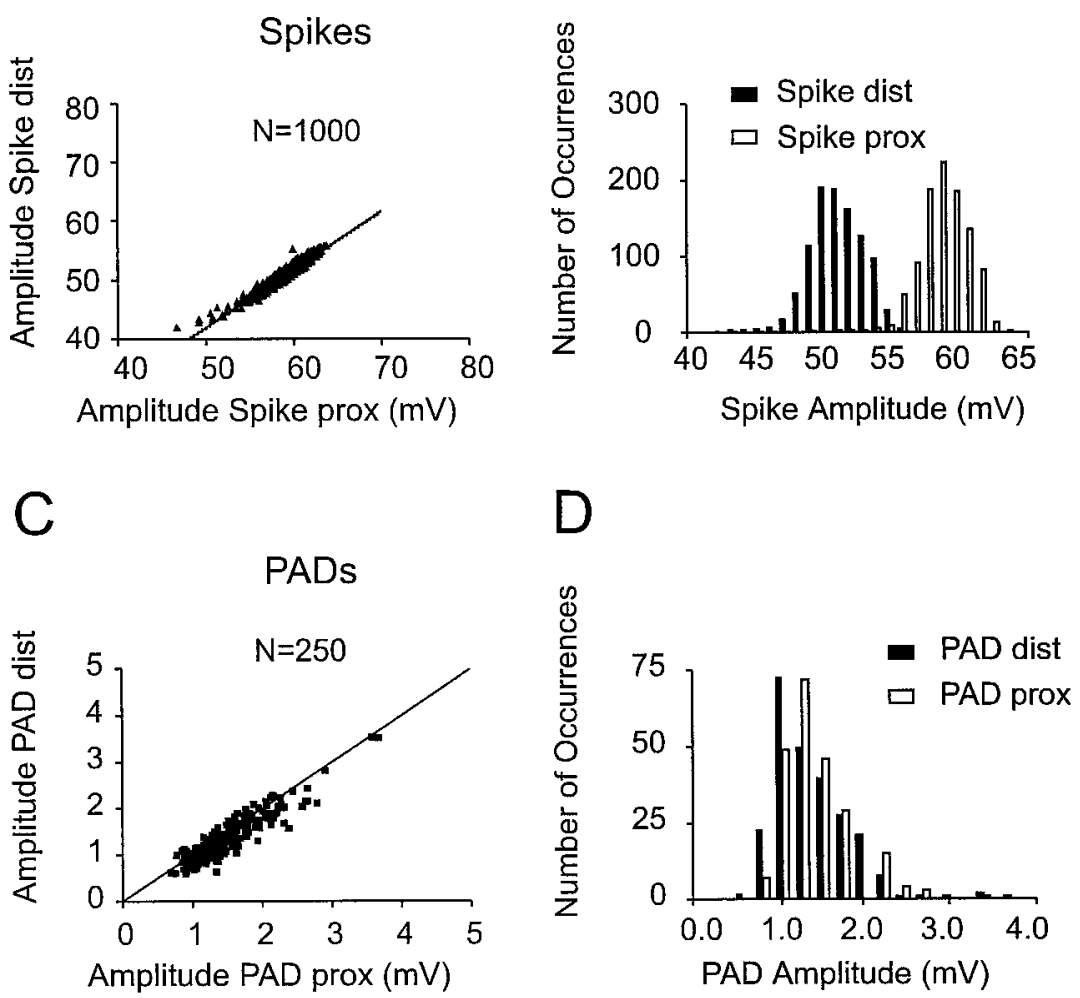

$E$

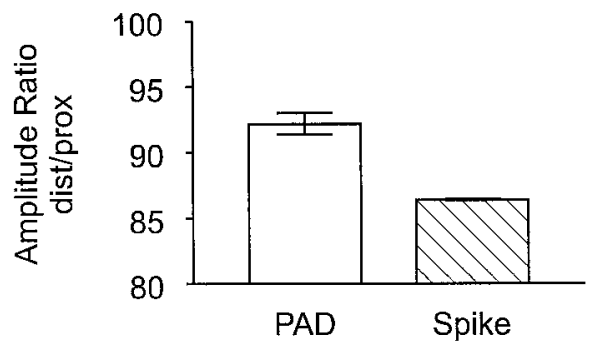

larized by $22 \mathrm{mV}$ (Fig. 9A). This effect could be caused by inactivation of $\mathrm{Na}^{+}$channels or an increased conductance that produced shunting of action potentials, or both. The input resistance decreased when the membrane potential was depolarized (Fig. 10D). The duration of this effect is $\sim 1-2 \mathrm{sec}$, which is three to five times larger than the duration of PADs. The membrane potential reached by the peak of action potentials remained unchanged when the membrane potential of CBTs was held between -90 and $-55 \mathrm{mV}$. On the other hand, at the resting potential, small PADs, which depolarized the membrane potential by $3-15 \mathrm{mV}$, produced a marked decrease of the spike peak (Fig. 10B). The effects of PADs on action potentials were compared with those of current injection (Fig. 10C). These results were consistent in the five experiments performed and suggest that the decrease of the peak of action potentials mediated by single PADs does not involve $\mathrm{Na}^{+}$channel inactivation but results from a shunting mechanism through increased chloride conductance.
Figure 7. Comparison of PAD and spike propagation in passive sites of $\mathrm{CBCO}$ axons: statistical analysis. The results are analyzed from the experiment shown in Figure 5. $A, B$, The spike amplitude at the distal recording site is always smaller than the proximal one. $A$, Graph plotting the amplitude of distal against proximal spike. The line is the linear regression curve $(r=0.93)$. B, Histograms of spike amplitudes recorded proximally (from ME1 in Fig. 5; open bars) and distally (from ME2 in Fig. 5; filled bars). $C, D$, The same analysis as in $A$ and $B$ but for the amplitude of PADs. $C$, Graph plotting distal against proximal PAD amplitude. The relationship is linear (dashed line represents the regression line; $r=$ $0.84)$. The continuous line represents the equation $y=x$. The fact that most of the points are below this line indicates that PADs display a smaller amplitude at the distal than at the proximal recording site. $D$, The distal and proximal PAD amplitude histograms largely overlap, the distal one being slightly displaced to the left. $E$, Graph of the amplitude ratio of PADs and spikes recorded at two sites in the same sensory axon.

\section{DISCUSSION}

PADs occur locally and shunt afferent action potentials Close apposition sites between GABA-ir and Lucifer yellowfilled axons (Fig. 2) were found in the area predicted by the electrophysiological analysis. With use of ultrastructural methods, axoaxonal synapses, which represent the morphological substrate of presynaptic inhibition, have been found in different species (Atwood and Morin, 1970; Nakajima et al., 1973; Hirosawa et al., 1981; Fyffe and Light, 1984; Wang-Bennett and Glantz, 1985; Watson et al., 1991; Lamotte d'Incamps et al., 1998a). Most inhibitory axoaxonal synapses, however, have been found close to presynaptic release sites (Atwood et al., 1984; Fyffe and Light, 1984; Lamotte d'Incamps et al., 1998a). Inhibitory axoaxonal synapses distant from output synapses have been described in the brain of crustacea (Wang-Bennett and Glantz, 1985). In this case, inhibitory axoaxonal GABAergic synapses are located on the terminal arbor between the axon and the output synapses. This 


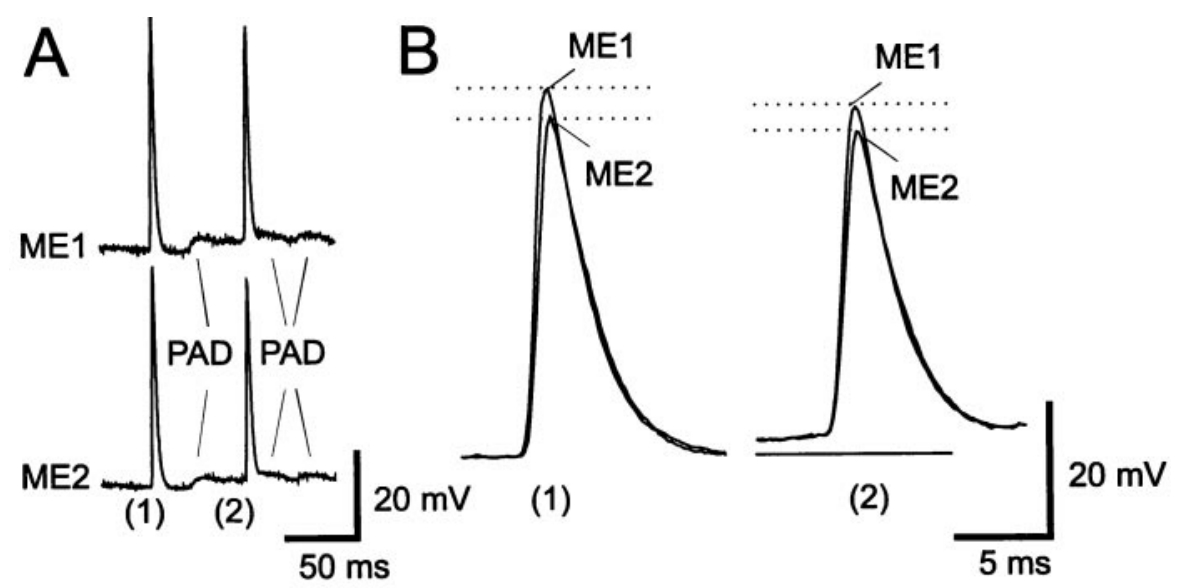

Figure 8. PADs reduce spike peaks at sites more proximal than ME1. $A$, Simultaneous intracellular recordings from ME1 and ME2 (same electrode positions as in Fig. 5). The first spike occurs in the absence of a PAD (1), whereas the second spike occurs in the presence of a PAD (2) and shows a reduced peak. $B$, Superimposed traces of proximal (ME1) and more distal (ME2) recordings from spike 1 (in the absence of PADs) and spike 2 (in the presence of PADs). In both the absence and presence of PADs, the amplitude of action potentials was reduced to $86 \%$ of the initial value because of the passive propagation between ME1 and ME2 recording sites, indicating that PADs did not produce any shunting of action potentials in the sensory axon between ME1 and ME2 recording sites. $C$, Schematic diagram representing the effect of the location of PADs on the amplitude of action potentials. If PADs occurs close to the first branching point $(C 1)$, the amplitude of action potential does not show any further decrease as they propagate from ME1 to ME2. If PADs occur more distal than the branching point, the amplitude of action potentials is decreased further $(C 2)$.
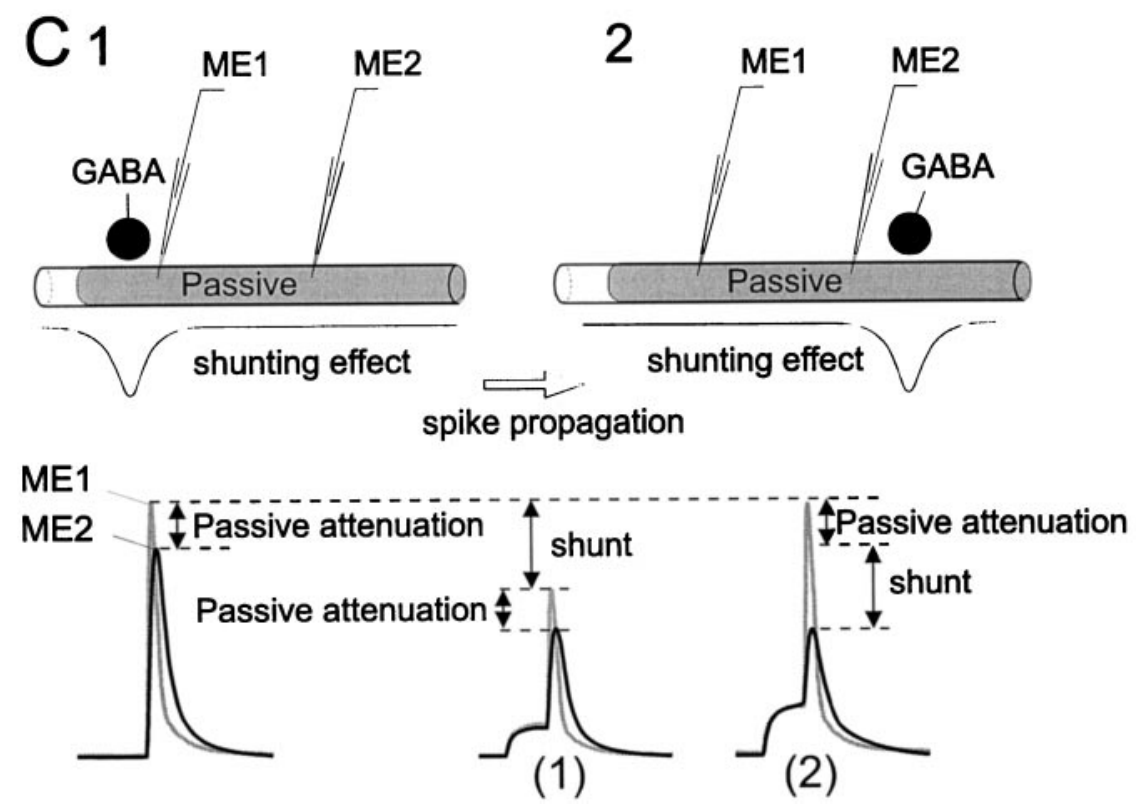

situation is more comparable to the CBTs. Confocal microscopy analysis suggests that such output synapses from CBT onto MNs are located after the branching point on the small branches (Fig. 3).

The fact that the inhibitory endings do not occur at or immediately adjacent to the release sites suggests that the inhibitory action does not directly act on the transmitter release machinery per se, but acts by decreasing the amplitude of action potential and thereby reducing transmitter release at the presynaptic terminals. This is supported by the electrophysiological findings reported in this study (Fig. 7). Because PADs did not produce any further reduction of action potentials in sites more distal to the first branching point, the GABA synapse responsible for PADs presumably acts before this first branching point, i.e., where the CBT enters the ganglion. We have previously reported the existence of a clear relationship between the amplitude of presynaptic spikes and that of postsynaptic EPSPs (Cattaert et al., 1992). The results of the present work indicate that the reduction of spike amplitude by PADs is likely to be directly responsible for the reduction of transmitter release. A direct effect onto the output synapse is unlikely to occur because GABA synapses are located far from the output synapses.

The electrophysiological and immunohistochemical analyses suggest that action potentials propagate passively after the first branching point and that GABAergic synapses mediating PADs are located close to the branching point. The increased chloride conductance during PADs will shunt afferent action potentials as they propagate toward the presynaptic terminals and thus reduce their efficiency in evoking transmitter release. The fact that the GABAergic inputs appear to be located primarily around the first branching point suggests that synaptic transmission from sensory neurons is globally controlled. GABAergic presynaptic inhibition does not seem to mediate selective control of transmitter release from different branches of single sensory axons. A local control of sensory information flow in different branches of single sensory axons has recently been shown in the cat spinal cord (Lomeli et al., 1998). In the crayfish, such a mechanism may involve other receptors or may be limited to some sensory axons. No presynaptic inhibition was observed in some CBTs (20\%) in which a specific filtering of afferent messages with respect to their target is therefore possible.

\section{Active and passive conduction in CBTs}

The results presented in this study suggest that only passive conduction occurs in the distal processes of CBTs; however, the precise location of this transition is difficult to determine on the basis of the electrophysiological analysis. Moreover, it is not 


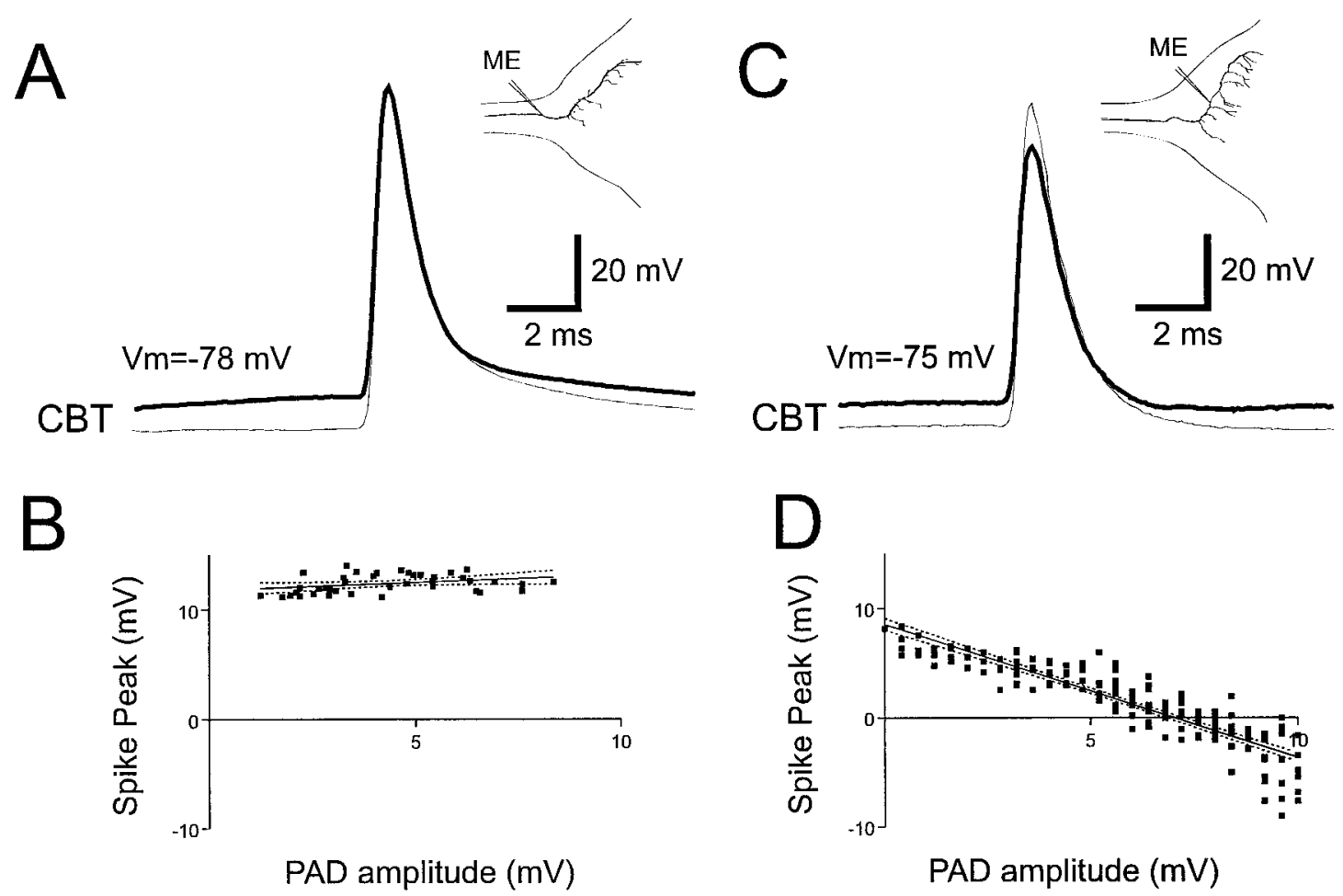

Figure 9. Effect of PADs on spike peaks at two locations of a CBCO sensory axon. $A$, Superimposed recordings from a site $100 \mu \mathrm{m}$ before the first branching point. Positioning of the microelectrode and anatomy of the sensory terminal (reconstructed after Lucifer yellow staining) are shown in the inset. The thin and thick traces were obtained in the absence and presence of a PAD, respectively. Note that peak values of both recordings are identical. $V_{\mathrm{m}}=$ resting membrane potential. $B$, Diagram of spike peak value against PAD amplitude. At this recording site, PADs have a very small but positive effect on the spike peak: the larger the PAD, the larger the spike. $C$, Superimposed recordings from a site $100 \mu \mathrm{m}$ after the first branching point. Positioning of the microelectrode and anatomy of the sensory terminal (reconstructed after Lucifer yellow staining) are shown in the inset. The thin and thick traces were again obtained in the absence and presence of a PAD, respectively. Note that the peak value in the presence of the PAD is reduced. $D$, Diagram of spike peak value against PAD amplitude. At this more distal recording site, PADs reduce spike peak linearly with respect to their amplitude. Recordings in $A$ and $C$ are from different experiments.

known whether the transition between the zone of active and passive conduction occurs gradually or abruptly. Results from simulation studies (our unpublished observations) have shown that the reduction of the orthodromic spike peak in the distal part of a CBT can be observed even if the recording is made from an active site. Therefore, the reduction of the spike peak per se is not an indication that we are recording from a passive site, but rather that the recording is made close to a passive zone. Such a

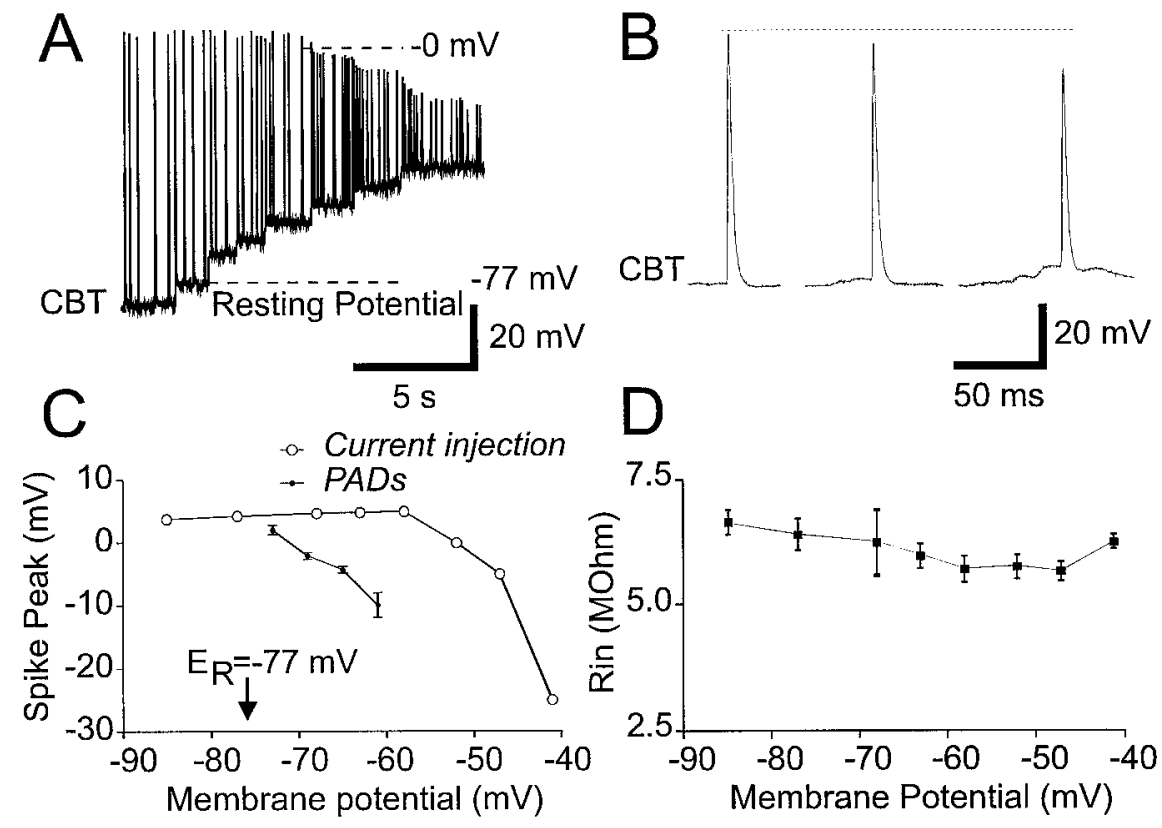

Figure 10. Shunting and inactivating mechanisms of PADs. $A$, Intracellular recording from a $\mathrm{CBCO}$ sensory axon. Two microelectrodes were placed in the same CBCO sensory axon. One was used to inject current steps (recording not shown), and the other was used to measure the voltage response. $B$, During the same experiment as in $A$, spontaneously occurring PADs reduced spike amplitudes. $C$, Comparison of the effects of spontaneous PADs and current injection on spike amplitude. The peak value is plotted against the membrane potential at the base of the spike. Although 1- to 17-mV-amplitude PADs (responsible for membrane potential at the base of spikes being in the range -77 to $-60 \mathrm{mV}$ ) induce a noticeable reduction of spike peak, the injection of depolarizing current does not induce any visible reduction of the peak until the membrane potential is increased to $-55 \mathrm{mV}$ and above (open symbols). $D$, Relationship between input resistance (Rin) and the imposed membrane potential during the experiment. 
reduction of spike peak is presented in Figure $4 B$, in which the more distal electrode is likely to be still in an active propagation zone. By comparison, purely passive propagation fulfills three criteria: spatial attenuation, low-pass filtering, and absence of any conduction delay. The latter criterion is often masked by the membrane potential noise. However, because this is the case when recording from sites $150 \mu \mathrm{m}$ more distal to the first branching point (Fig. 5), the passive propagation zone is likely to start $\sim 150 \mu \mathrm{m}$ after the first branching point.

\section{Shunting mechanism versus inactivation of $\mathrm{Na}^{+}$channels}

We have shown previously that PADs result from the opening of a GABA-activated receptor channel (Cattaert et al., 1992) and that local application of GABA depolarizes the membrane potential of CBTs by $\sim 20 \mathrm{mV}$ and dramatically reduces the spike amplitude by $75 \%$, because of a decrease of input resistance by up to $67 \%$. This decrease in input resistance is probably responsible for most of the reduction of spike amplitude. In this report, we have demonstrated that for small PADs $(<20 \mathrm{mV})$ only a shunting mechanism would be involved (Fig. 10). Larger PADs, such as those occurring during locomotor-related bursts, could possibly reduce the peak of orthodromic spikes by inactivating $\mathrm{Na}^{+}$channels. We were unable to obtain the inactivation curve for $\mathrm{Na}^{+}$in these axons because the CBTs cannot be clamped because of their extensive branching. However, an argument against such an inactivation process is that large PADs often elicit antidromic spikes (El Manira et al., 1991; Cattaert et al., 1992, 1994). This apparent contradiction is enhanced by the fact that such antidromic spikes do not elicit any EPSP in postsynaptic MNs (Cattaert et al., 1992). One would think that if inactivation played the major role in presynaptic inhibition, such antidromic spikes would produce EPSPs in the postsynaptic neurons, as do orthodromic spikes; however, this is not the case. A simulation study has demonstrated that the reduction of spike peak largely depends on where inactivation of $\mathrm{Na}^{+}$channels is produced relatively to the passive zone (our unpublished observations). Spikes produced near the transition between active and passive zones by large PADs will not be propagated in both directions. However, in this situation, the shunting mechanism is largely involved in preventing spikes being conveyed toward the terminal endings (our unpublished observations). These simulation studies suggest that in this case spikes are likely to be triggered in sites situated more proximally to GABA synapses because at the GABA synapse site, as local currents leak through the chloride channels, the spike is prevented from developing fully. Such mechanisms must also exist in mammals because antidromic discharges are produced during fictive locomotion in the cat (Dubuc et al., 1988; Gossard et al., 1989). However, in this case, PADs are supposed to be produced close to the output synapse (Fyffe and Light, 1984). In the latter case, the question remains whether antidromic discharges produced by PADs would produce an EPSP.

Our conclusion emphasizes the role of a shunting mechanism in PAD-mediated presynaptic inhibition. This finding is different from previous simulation studies in mammals (Graham and Redman, 1994; Walmsley et al., 1995; Lamotte d'Incamps et al., 1998a,b). Simulation studies, based on serial electronic microscopy reconstruction of Ia sensory neurons (Walmsley et al., 1995), demonstrated that inactivation could play the main role in presynaptic inhibition. This conclusion was supported by several data. First, input synapses (likely GABAergic) are located at some distance from the terminal but are too few to produce a massive shunting. Second, PADs propagate easily in the branches and may inactivate the incoming spike at some distance from the GABA synapse. Recent studies in cat confirm this hypothesis (B. Lamotte d'Incamps, unpublished observations).

Our results do not totally contradict these findings because, as we proposed, both mechanisms (shunting and inactivation) are present, depending on the PAD amplitude. Our electrophysiological data (Fig. 10) demonstrate that small PADs $(<10 \mathrm{mV})$ produce exclusively shunting, whereas larger PADs such as those occurring during phasic bursts during locomotion (up to $25 \mathrm{mV}$ ) will also induce inactivation of $\mathrm{Na}^{+}$channels. An important point concerns the resting potential of the sensory terminal. In the crayfish it is generally around $-75 \mathrm{mV}$. Therefore small PADs cannot inactivate $\mathrm{Na}^{+}$channels. Another point concerns the number of GABA synapses and the amount of shunting they produce. In the crayfish $\mathrm{CBCO}$ terminals, we have demonstrated that a large shunt (67\% decrease in input resistance) can be produced when most of the GABA synapses are activated by local application of GABA via a pressure ejection micropipette (Cattaert et al., 1992). This finding was supported in the present work by the demonstration that GABA-ir boutons were found concentrated in the region of the first branching point. Although an ultrastructural analysis is required before it can be concluded that these boutons represent GABA synapses, the existence of so many GABAergic synapses at that site is in accordance with the possibility of a shunting mechanism.

\section{REFERENCES}

Allen T, Burnstock G (1990) $\mathrm{GABA}_{\mathrm{A}}$ receptor-mediated increase in membrane chloride conductance in rat paratracheal neurones. $\mathrm{Br} \mathrm{J}$ Pharmacol 100:261-268.

Atwood HL, Morin WA (1970) Neuromuscular and axoaxonal synapses of the crayfish opener muscle. J Ultrastruct Res 32:351-369.

Atwood HL, Stevens JK, Marin L (1984) Axo-axonal synapse location and consequences for presynaptic inhibition in crustacean motor axon terminals. J Comp Neurol 225:64-74.

Burrows M, Matheson T (1994) A presynaptic gain control mechanism among sensory neurons of a locust leg proprioceptor. J Neurosci $14: 272-282$.

Cattaert D, El Manira A, Clarac F (1992) Direct evidence for presynaptic inhibitory mechanisms in crayfish sensory afferents. J Neurophysiol 67:610-624.

Cattaert D, El Manira A, Clarac F (1994) Chloride conductance produces both presynaptic inhibition and antidromic spikes in primary afferents. Brain Res 666:109-112.

Chrachri A, Clarac F (1989) Synaptic connections between motor neurons and interneurons in the fourth thoracic ganglion of the crayfish Procambarus clarkii. J Neurophysiol 62:1237-1250.

Clarac F, El Manira A, Cattaert D (1992) Presynaptic control as a mechanism of sensory-motor integration. Curr Opin Neurobiol 2:764-769.

Dubuc R, Cabelguen J-M, Rossignol S (1988) Rhythmic fluctuations of dorsal root potentials and antidromic discharges of primary afferents during fictive locomotion in the cat. J Neurophysiol 60:2014-2036.

El Manira A, Clarac F (1991) GABA-mediated presynaptic inhibition in crayfish primary afferents by non-A, non-B GABA receptors. Eur J Neurosci 3:1208-1218.

El Manira A, DiCaprio RA, Cattaert D, Clarac F (1991) Monosynaptic interjoint reflexes and their central modulation during fictive locomotion in crayfish. Eur J Neurosci 3:1219-1231.

Fyffe RE, Light AR (1984) The ultrastructure of group Ia afferent fiber synapses in the lumbosacral spinal cord of the cat. Brain Res 300:201-209.

Gossard J-P, Cabelguen J-M, Rossignol S (1989) Intra-axonal recordings of cutaneous primary afferents during fictive locomotion in the cat. J Neurophysiol 62:1177-1188.

Gossard J-P, Cabelguen J-M, Rossignol S (1990) Phase-dependent mod- 
ulation of primary afferent depolarization in single cutaneous primary afferents evoked by peripheral stimulation during fictive locomotion in the cat. Brain Res 537:14-23.

Graham B, Redman SA (1994) Simulation of action potentials in synaptic boutons during presynaptic inhibition. J Neurophysiol 71:538-549.

Hirosawa K, Tao-Cheng J-H, Nakajima Y, Tisdale AD (1981) Thinsection and freeze-fracture studies of crayfish stretch receptor synapses including the reciprocal inhibitory synapse. J Comp Neurol 200:39-53.

Jimenez I, Rudomin P, Solodkin M (1988) PAD patterns of physiologically identified afferent fibers from the medial gastrocnemius muscle. Exp Brain Res 71:643-657.

Lamotte d'Incamps B, Destombes J, Thiesson D, Hellio R, Lasserre X, Kouchtir-Devanne N, Jami L, Zytnicki D (1998a) Indications for GABA-immunoreactive axo-axonic contacts on the intraspinal arborization of a Ib fiber in cat: a confocal microscope study. J Neurosci 18:10030-10036.

Lamotte d'Incamps B, Meunier C, Monnet M-J, Jami L, Zytnicki D (1998b) Reduction of presynaptic action potentials by PAD: model and experimental study. J Comput Neurosci 5:141-156.

Lomeli J, Quevedo J, Linares P, Rudomin P (1998) Local control of information flow in segmental and ascending collaterals of single afferents. Nature 395:600-604.

Maxwell DJ, Christie WM, Short AD, Brown AG (1990) Direct observations of synapses between GABA-immunoreactive boutons and muscle afferent terminals in lamina VI of the cat's spinal cord. Brain Res 530:215-222.
Nakajima Y, Tisdale AD, Henkart MP (1973) Presynaptic inhibition at inhibitory nerve terminals: a new synapse in the crayfish stretch receptor. Proc Natl Acad Sci USA 70:2462-2466.

Nusbaum MP, El Manira A, Gossard JP, Rossignol S (1997) Presynaptic mechanisms during rhythmic activity in vertebrates and invertebrates. In: Neurons, networks, and motor behavior (Stein PSG, Grillner S, Selverston AI, Stuart DG, eds), pp 237-253. Cambridge, MA: MIT.

Rudomin P, Romo R, Mendell LM (1998) Presynaptic inhibition and neural network. New York: Oxford UP.

Segev I (1990) Computer study of presynaptic inhibition controlling the spread of action potentials into axon terminals. J Neurophysiol 63:987-998.

Sillar KT, Skorupski P (1986) Central input to primary afferent neurons in crayfish, Pacifastacus leniusculus, is correlated with rhythmic motor output of thoracic ganglia. J Neurophysiol 55:678-688.

Walmsley B, Graham B, Nicol MJ (1995) Serial E-M and simulation study of presynaptic inhibition along a group Ia collateral in the spinal cord. J Neurophysiol 74:616-623.

Wang-Bennett LT, Glantz RM (1985) Presynaptic inhibition in the crayfish brain. II. Morphology and ultrastructure of the terminal arborization. J Comp Physiol [A] 156:605-617.

Watson AHD, Storm-Mathisen J, Ottersen OP (1991) GABA and glutamate-like immunoreactivity in processes presynaptic to afferents from hair plates on the proximal joints of the locust leg. J Neurocytol 20:796-809. 